\title{
A new application of microwave technology to proteomics
}

\author{
Hsueh-Fen Juan ${ }^{1,2}$, Shing-Chuan Chang ${ }^{2}$, Hsuan-Cheng Huang ${ }^{3}$ and Shui-Tein Chen ${ }^{4,5}$ \\ ${ }^{1}$ Institute of Molecular and Cellular Biology, National Taiwan University, Taipei \\ ${ }^{2}$ National Taipei University of Technology, Taipei \\ ${ }^{3}$ Institute of Bioinformatics, National Yang-Ming University, Taipei \\ ${ }^{4}$ Institute of Biological Chemistry, Academia Sinica, Taipei \\ ${ }^{5}$ Institute of Biochemical Sciences, National Taiwan University, Taipei \\ Taiwan
}

Two-dimensional electrophoresis (2-DE) combined with mass spectrometry has significantly improved the possibilities of large-scale identification of proteins. However, 2-DE is limited by its inability to speed up the in-gel digestion process. We have developed a new approach to speed up the protein identification process utilizing microwave technology. Proteins excised from gels are subjected to in-gel digestion with endoprotease trypsin by microwave irradiation, which rapidly produces peptide fragments. The peptide fragments were further analyzed by matrix-assisted laser desorption/ionization technique for protein identification. The efficacy of this technique for protein mapping was demonstrated by the mass spectral analyses of the peptide fragmentation of several proteins, including lysozyme, albumin, conalbumin, and ribonuclease A. The method reduced the required time for in-gel digestion of proteins from 16 hours to as little as five minutes. This new application of microwave technology to protein identification will be an important advancement in biotechnology and proteome research.

\section{Keywords:}

In-gel digestion / Microwave

Proteomics characterizes cellular proteins and their abundance, state of modification, protein complexes and interactions [1]. The global changes in cellular protein expression can be visualized by 1-D or 2-DE and identified by MS [2]. Peptide identification can be accomplished by peptide mass mapping. The first step is the in-gel digestion of proteins by sequencespecific proteases. Since each amino acid residue has a unique mass, protein digestion will yield a set of distinct peptides specific to each protein. A mass spectrum of digested peptides, therefore, results in a unique PMF. The set of peptide masses obtained by MS is then used to search against protein databases created by in silico cleavage of all known sequences [3]. This method has been shown to be particularly successful for the identification of proteins [1, 4-6]. However, it is limited by its inability to speed up the in-gel digestion process.

Correspondence: Dr. Hsueh-Fen Juan, Department of Life Science, Institute of Molecular and Cellular Biology, National Taiwan University, No. 1, Sec. 4, Roosevelt Road, Taipei, 106 Taiwan E-mail: yukijuan@ntu.edu.tw

Fax: +886-2-23673374
Received: May 29, 2004

Revised: September 5, 2004

Accepted: October 1, 2004
Microwave applications in peptide biochemistry [7], immunohistochemistry [8], antigen retrieval [9], protein staining techniques [10], DNA extraction method [11], and enzyme reaction [12] have been reported. However, the application of microwave to in-gel digestion has not been previously described in detail in the literature despite its apparent use in laboratories.

In this report, we describe a new approach utilizing microwave technology to speed up the in-gel digestion reaction. Proteins excised from gels are subjected to in-gel digestion with endoprotease trypsin under microwave irradiation instead of conventional incubation at $37^{\circ} \mathrm{C}$. Furthermore, the digested peptide fragments were analyzed by MALDI-quadrupole(Q)-TOF MS for protein identification using both PMF and MS/MS technique. First, five proteins, including lysozyme, albumin from chicken egg, albumin from bovine, conalbumin, and ribonuclease were separated by 1-D SDS-PAGE and stained by Coomassie blue (Fig. 1). The Coomassie blue-stained protein bands were excised from the gel and digested with trypsin (Promega, Madison, WI, USA). The gel pieces were soaked in $100 \%$ acetonitrile 


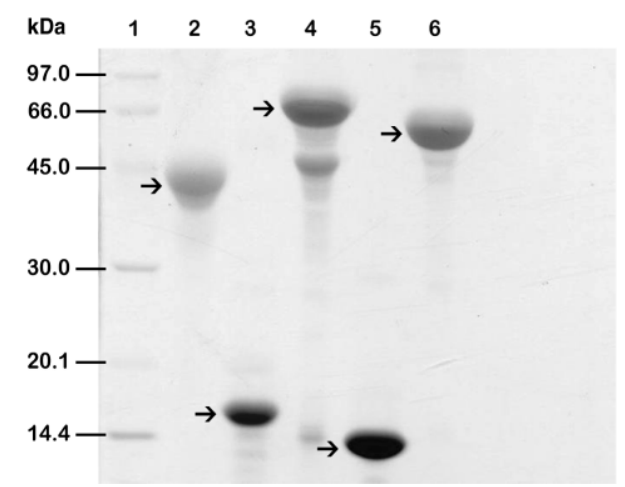

Figure 1. 1-DE separation of five proteins ( $25 \mu \mathrm{g}$ per protein) in $12.5 \%$ SDS gel. The gel was stained with Coomassie blue. Lane 1, molecular weight markers; lane 2, albumin (chicken egg); lane 3, ribonuclease A; lane 4, conalbumin; lane 5, lysozyme; lane 6, albumin (bovine). Protein bands marked by black arrows were further in-gel digested by the traditional or microwave method and identified by mass spectrometry.

for $5 \mathrm{~min}$, dried in a lyophilizer for 20-30 min and rehydrated in $25 \mathrm{~mm}$ ammonium bicarbonate buffer ( $\mathrm{pH} 8.0$ ) containing $35 \mu \mathrm{L}$ of $10 \mu \mathrm{g} / \mathrm{mL}$ trypsin until the gel pieces were fully immersed. The gel solution with trypsin was put into a microwave for $5 \mathrm{~min}$ at $195 \mathrm{~W}$ or $325 \mathrm{~W}$, incubated for $5 \mathrm{~min}$ or $16 \mathrm{~h}$ at $37^{\circ} \mathrm{C}$. After the in-gel digestion either with or without microwave irradiation, the peptide fragments were extracted twice with $50 \mu \mathrm{L}$ of $50 \%$ acetonitrile/0.1\% TFA. After removal of acetonitrile by centrifugation in a vacuum centrifuge, the peptides were directly spotted on the sample plate of the MALDI-TOF mass spectrometer. Finally, CHCA $(0.5 \mu \mathrm{L}$ of $10 \mathrm{mg} / \mathrm{mL})$ was applied to each spot, and the spots were air-dried at room temperature prior to acquiring mass spectra (M@LDI ${ }^{\mathrm{T}}$, Micromass, Manchester, UK). The mass spectrum of tryptic peptides of albumin (chicken egg) are shown in Fig. 2A for the traditional method and Fig. 2B for the microwave method.

The monoisotopic peptide mass values were searched against the Swiss-Prot database using the MASCOT PMF search program (http://www.matrixscience.com) [13]. The corresponding matched tryptic fragments versus theoretical tryptic fragments using PMF are shown in Table 1. A larger number of matched tryptic fragments implies higher yields of proteolytic digestion. The method using microwave power of $195 \mathrm{~W}$ gives more matched fragments than the traditional method $\left(37^{\circ} \mathrm{C}, 16 \mathrm{~h}\right)$ for all the proteins except conalbumin; in this case the number of matched fragments is slightly lower but close to the traditional method. Considering the required reaction time for the traditional method is much longer, the microwave method apparently gives higher efficiency for in-gel digestion. Besides using PMF, the most intense ions in the TOF-MS spectrum were selected to perform an optimized MS/MS analysis. The product ion spectra generated by MALDI-TOF-MS/MS were searched against the Swiss-Prot database for exact matches using the MASCOT MS/MS ion search program [13]. All five protein bands were
Table 1. The comparison of the MALDI-TOF-MS and MS/MS data for five standard proteins using traditional and microwave methods

\begin{tabular}{|c|c|c|c|c|c|}
\hline \multirow[b]{2}{*}{ Power } & & \multicolumn{2}{|c|}{ Traditional $\left(37^{\circ} \mathrm{C}\right)$} & \multicolumn{2}{|c|}{ Microwave } \\
\hline & & & & $195 \mathrm{~W}$ & $325 \mathrm{~W}$ \\
\hline Time & & $16 \mathrm{~h}$ & $5 \min$ & $5 \mathrm{~min}$ & $5 \min$ \\
\hline \multirow{3}{*}{$\begin{array}{l}\text { Albumin } \\
\quad \text { (chicken egg) }\end{array}$} & $\mathrm{P} / \mathrm{T}^{\mathrm{a})}$ & $9 / 33$ & $0 / 33$ & $11 / 33$ & $8 / 33$ \\
\hline & PMF score ${ }^{\text {b) }}$ & 60 & - e) & 73 & 45 \\
\hline & MS/MS score ${ }^{c)}$ & 45 & - & 60 & 38 \\
\hline \multirow[t]{3}{*}{ Ribonuclease A } & $\mathrm{P} / \mathrm{T}$ & $0 / 15$ & $0 / 15$ & $6 / 15$ & $9 / 15$ \\
\hline & PMF score & - & - & 46 & 60 \\
\hline & MS/MS score & 70 & - & 163 & 83 \\
\hline \multirow[t]{3}{*}{ Lysozyme } & $\mathrm{P} / \mathrm{T}$ & $6 / 18$ & $0 / 18$ & $10 / 18$ & $10 / 18$ \\
\hline & PMF score & 52 & - & 61 & 63 \\
\hline & MS/MS score & 70 & - & 173 & 125 \\
\hline \multirow[t]{3}{*}{ Conalbumin } & $\mathrm{P} / \mathrm{T}$ & $20 / 87$ & $0 / 87$ & $18 / 87$ & $19 / 87$ \\
\hline & PMF score & 69 & - & 57 & 56 \\
\hline & MS/MS score & $13^{\mathrm{d})}$ & - & 150 & 131 \\
\hline \multirow{3}{*}{$\begin{array}{l}\text { Albumin } \\
\text { (bovine) }\end{array}$} & $\mathrm{P} / \mathrm{T}$ & $9 / 78$ & $0 / 78$ & $17 / 78$ & $15 / 78$ \\
\hline & PMF score & 35 & - & 41 & 39 \\
\hline & MS/MS score & 90 & - & 174 & $29^{\mathrm{d})}$ \\
\hline
\end{tabular}

a) $\mathrm{P} / \mathrm{T}$ denotes the number of matched peptide fragments versus the theoretical number of total peptide fragments by trypsin in PMF analysis

b) The probability-based MOWSE score returned from the MASCOT peptide mass fingerprint search program

c) The probability-based MOWSE score returned from the MASCOT MS/MS ion search program

d) The produced ion spectra failed to be identified as the correct proteins

e) Not determined

successfully identified as albumin from chicken egg, ribonuclease A, conalbumin, lysozyme, and albumin from bovine, respectively. The corresponding search scores are shown in Table 1. The method using microwave power of $195 \mathrm{~W}$ gives the highest scores. In conclusion, we have shown that microwave-assisted reactions can produce high efficiency, purity and accuracy in minutes, while traditional methods would require hours.

We have successfully demonstrated the usefulness of a new method to apply microwave to in-gel digestion and to combine with MALDI-Q-TOF for protein identification. This approach speeds up the in-gel digestion process to minutes versus hours using traditional methods. The use of microwave technology in protein identification will be a very powerful tool in proteome research and may prove successful in drug discovery and development.

This work was supported by National Science Council of Taiwan (NSC 93-3112-B-002-042). We gratefully acknowledge the Core Facilities for Proteomics Research, Academia Sinica, Taiwan and Supachai Topanurak for technical support. 


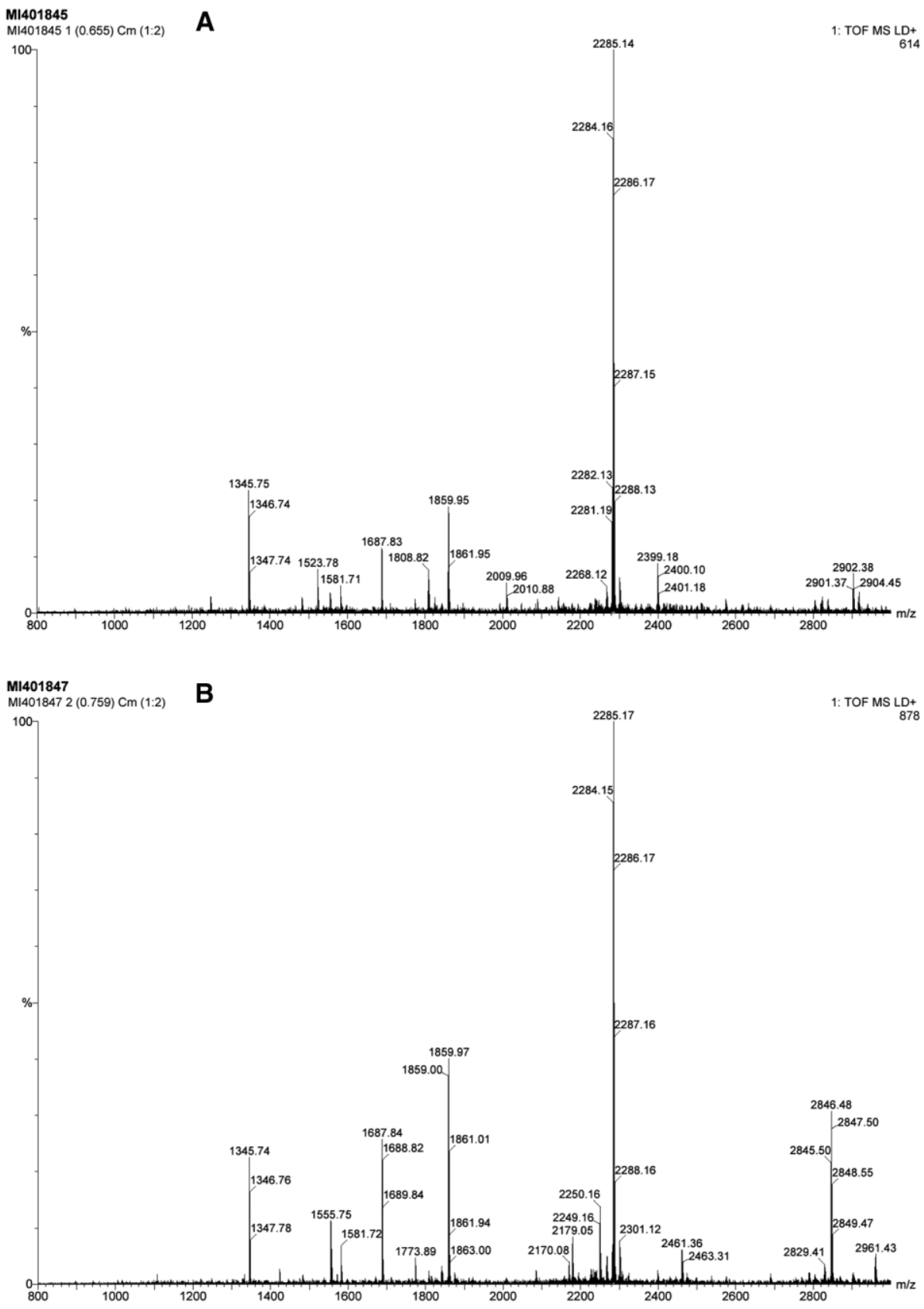

Figure 2. The MALDI-TOF mass spectrum of albumin (chicken egg) is shown. The mass spectrum was obtained after in-gel digestion: (A) using the traditional method, incubated for $16 \mathrm{~h}$ at $37^{\circ} \mathrm{C}$; (B) using the microwave method with microwave irradiation of $195 \mathrm{~W}$ for 5 min.

\section{References}

[1] Gygi, S. P., Aebersold, R., Curr. Opin. Chem. Biol. 2000, 4, 489494.

[2] Sinchaikul, S., Sookkheo, B., Topanuruk, S., Juan, H. F. et al., J. Chromatogr. B 2002, 771, 261-287.

[3] Fenyo, D., Curr. Opin. Biotechnol. 2000, 11, 391-395.

[4] Yates, J. R. III, Trends Genet 2000, 16, 5-8.

[5] Patterson, S. D., Aebersold, R., Electrophoresis 1995, 16, 1791-1814.

[6] Juan, H. F., Lin, J. Y. C., Chang, W. H., Wu, C. Y. et al., Electrophoresis 2002, 23, 2490-2504.
[7] Chen, S. T., Chiou, S. H., Wang, K. T., J. Chin. Chem. Soc. 1991, 38, 85-91.

[8] Schad, A., Fahimi, H. D., Volkl, A., Baumgart, E., J. Histochem. Cytochem. 2003, 51, 751-760.

[9] Redkar, A. A., Krishan, A., Cytometry 1999, 38, 61-69.

[10] Nesatyy, V. J., Dacanay, A., Kelly, J. F., Ross, N. W., Rapid Commun. Mass Spectrom. 2002, 16, 272-280.

[11] Sato, Y., Sugie, R., Tsuchiya, B., Kameya, T. et al., Diagn. Mol. Pathol. 2001, 10, 265-271.

[12] Pramanik, B. N., Mirza, U. A., Ing, Y. H., Liu, Y. H. et al., Protein Sci. 2002, 11, 2676-2687.

[13] Hirosawa, M., Hoshida, M., Ishikawa, M., Toya, T., Comput. Appl. Biosci. 1993, 9, 161-167. 